\title{
Un orologio solare miniaturistico in avorio da un contesto residenziale tardo-repubblicano di Pisa
}

\author{
Emanuele Taccola ${ }^{1}$, Jérôme Bonnin ${ }^{2}$, Denis Savoie ${ }^{3}$, e Filippo Battistoni ${ }^{4}$ \\ ${ }^{1}$ Università di Pisa <emanuele.taccola@unipi.it> \\ ${ }^{2}$ Université de Lille $3<$ jeromebonnin@laposte.net> \\ ${ }^{3}$ Syrte, Observatoire de Paris, Universcience < denis.savoie@universcience.fr> \\ ${ }^{4}$ Università di Pisa <filippo.battistoni@unipi.it>
}

\begin{abstract}
The object presented in this contribution adds to the relatively small corpus of GrecoRoman miniature sundials. It is a conical sundial made of elephant ivory, found in secondary deposition within an Augustan-age residential context in the Piazza del Duomo in Pisa, Italy. The article provides a thorough gnomonic study for this unique sundial as well as a detailed appreciation of related cultural, social, and typological aspects. This discovery may help to better define the social level of this area of the city between 2nd and 1st c. BCE.
\end{abstract}

\begin{abstract}
L'oggetto che si presenta in questo contributo costituisce un'integrazione al corpus relativamente poco cospicuo di orologi solari miniaturistici greco-romani. Si tratta di una meridiana conica in avorio di elefante, rinvenuta in giacitura secondaria all'interno di un contesto residenziale di età augustea nella Piazza del Duomo di Pisa. Al di là dell'unicità dell'esemplare, per il quale si fornisce un accurato studio gnomonico, unitamente a un approfondito apprezzamento dei relativi aspetti culturali, sociali e tipologici, tale scoperta contribuisce a meglio definire il livello sociale di quest'area della città tra il II e il I sec. a.C.
\end{abstract}

Keywords: Sundial, ivory, Graeco-Roman science, Late Republican Pisa, social time

Parole chiave: Orologio solare, avorio, scienza greco-romana, Pisa tardo-repubblicana, tempo sociale

In un recente convegno dedicato all'architettura residenziale di Pisa in età romana e medievale ${ }^{1}$ è stata resa nota un'inedita sequenza di domus databili tra l'età tardo-repubblicana e la fine del I sec. a.C., ${ }^{2}$ ubicate nell'attuale piazza del Duomo, a pochi metri in direzione est della Torre pendente (Fig. 1). ${ }^{3}$

L'identificazione dei lacerti murari e la loro associazione ad ambienti di tipo abitativo domestico è stata possibile in seguito a un faticoso lavoro di ricostruzione stratigrafica e analisi del materiale archeologico mobile di uno scavo ivi condotto negli anni 1985-88 (saggio D) e recentemente pubblicato da chi scrive. ${ }^{4}$

L'occasione ha reso necessario esaminare in maniera più scrupolosa i materiali dei livelli afferenti alla prima e media età augustea: tra i resti faunistici, ancora ricoperti della terra di scavo, si distinguevano quattro frammenti di forma anomala, ovvero troppo regolare rispetto alle ossa animali residuo di pasto o macellazione. L'intervento conservativo che ne

$1 \quad$ Cantini et al. 2020.

2 Taccola 2020.

3 Il saggio è ubicato nell'area compresa tra il recinto settentrionale del giardino del Museo dell'Opera e la strada che attraversa la piazza in senso est-ovest fino all'abside del duomo.

4 Taccola 2019.

(C) The Author(s), 2021. Published by Cambridge University Press. This is an Open Access article, distributed under the terms of the Creative Commons Attribution licence (http://creativecommons.org/licenses/by/4.0/), which permits unrestricted re-use, 


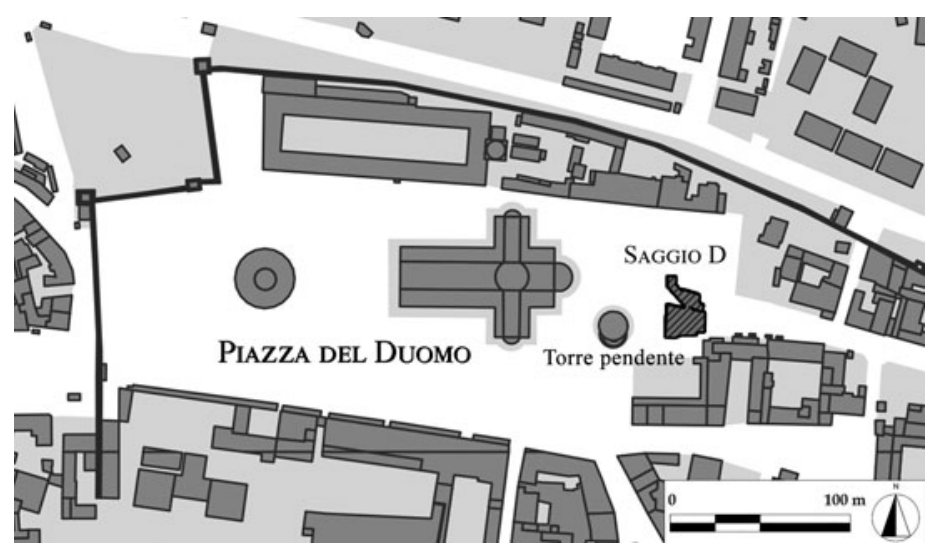

Fig. 1. Posizionamento dello scavo del settore orientale di piazza del Duomo (saggio D). (E. Taccola.)

è seguito ${ }^{5}$ ha consentito la ricomposizione di un oggetto inedito nel panorama archeologico dell'Italia antica (benché già noto tipologicamente): un orologio solare miniaturistico in avorio. ${ }^{6}$

\section{Il contesto di rinvenimento}

Il quadrante è stato rinvenuto in un livello riconducibile allo scorcio del I sec. a.C., più precisamente nella fossa di fondazione di un condotto fognario ${ }^{7}$ che percorreva in senso nord-sud un asse stradale su cui si affacciavano gli edifici di età augustea (Fig. 2). ${ }^{8}$

Ai materiali già indicati in altre sedi ${ }^{9}$ per datare la messa in opera e l'uso di questa infrastruttura (una piastra di tegola con bollo $\mathrm{V}^{\wedge} \mathrm{O}^{\wedge} \mathrm{L}^{\wedge} \mathrm{U}^{\wedge} \mathrm{Z}$, monogrammato e con ductus destrorso di tipo $2,{ }^{10}$ riferibile a una produzione etrusco-settentrionale certamente avviata nel corso della prima metà del I sec. a.C., ma forse già dalla fine del II sec. a.C.,

5 Il restauro e consolidamento del manufatto è stato eseguito da Gianluca Buonomini del Laboratorio di Disegno e Restauro (Dipartimento di Civiltà e Forme del Sapere, Università di Pisa).

6 Sebbene siano noti orologi solari miniaturistici in Italia, concentrati a Siracusa, nessuno di questi è realizzato in avorio: per un elenco più dettagliato, vd. sotto, "I quadranti miniaturistici nel corpus gnomonico antico" e Tabella 1 .

7 US 118 "riempimento della fossa praticata per la messa in opera della fogna" (scavi 1985, quadrato D 1).

8 Taccola 2019, 19. L'occupazione di questo settore di Pisa è tuttavia ancora più antica, risalendo almeno alla seconda metà del VI secolo a.C. Fino allo scorcio del III secolo a.C. le strutture e gli apprestamenti portati alla luce e la cultura materiale recuperata convergono nell'identificare il sito come un'area a destinazione santuariale. A partire dal secondo quarto del II secolo a.C. gli interventi edilizi riconosciuti stratigraficamente sembrano rispondere a un progetto urbanistico organico, che rimodula la fisionomia di questo settore della città antica, soprattutto dal punto di vista della destinazione d'uso dello spazio. Infatti, il sito, da area deputata a ospitare strutture e attività presumibilmente connesse alla sfera sacra, assume una connotazione di tipo residenziale che manterrà - con varie fasi edilizie - fino a età tardo-antica (Taccola 2019, 17, 27-37 e 250-55 con bibl. precedente a n. 838).

9 Taccola 2020, 26, fig. 11.

10 Gliozzo, Manacorda e Shepherd 2004, 195: in questa sede è segnalato un bollo da Pisa, attualmente irreperibile. All'esemplare dal saggio D si aggiunge una terza attestazione da via della Faggiola, per cui cfr. Rizzitelli e Fabiani 2020, 45, fig. 12. Per la datazione di questa categoria 


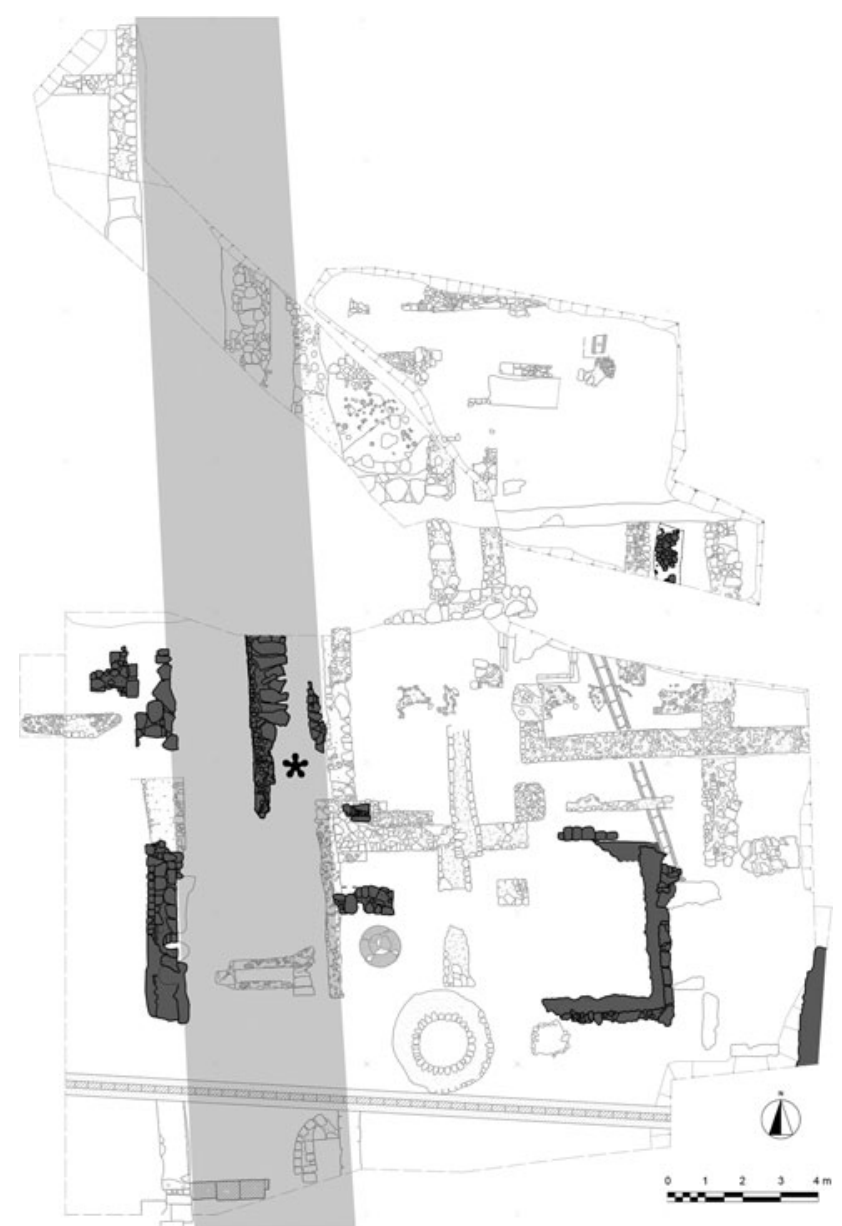

Fig. 2. Planimetria generale dello scavo del settore orientale di piazza del Duomo (saggio D). In grigio scuro le strutture di età augustea; in grigio chiaro l'asse stradale nord-sud percorso dal condotto fognario. L'asterisco indica il luogo di rinvenimento dell'orologio solare. (E. Taccola.) e un fondo di coppa di sigillata italica con bollo centrale in cartiglio rettangolare prodotta dall'officina pisana di Xanthus, databile genericamente tra 5 a.C. e $50 \mathrm{~d}$. C.), ${ }^{11}$ si sommano altri due reperti che contribuiscono a circostanziarne cronologicamente la fondazione: ${ }^{12}$ il primo è un frammento di sigillata italica con bollo centrale Atei in cartiglio rettangolare, datato tra il 15 a.C. e il 30 d.C. ${ }^{13}$ il secondo è un asse di bronzo di Augusto coniato da Marcus Maecinius Tullus, triumviro monetario per l'anno 7 a.C., ${ }^{14}$ che a nostro avviso costituisce il terminus post quem decisivo per la datazione dell'impianto della fognatura (Fig. 3).

\section{Descrizione dell'orologio}

Si tratta di un orologio conico, appartenente alla tipologia "Vertex Above the Horizontal Top Surface - South Facing Conical Dials" di Sharon L. Gibbs. ${ }^{15}$ Questa categoria di quadranti solari, tra i più diffusi in antichità, era definita nel mondo greco-romano $\kappa \hat{\omega} v o s$ o conus. ${ }^{16}$ Secondo Vitruvio 9.8, la

di oggetti e la localizzazione delle figlinae delle tegole con bollo tipo 2, cfr. Genovesi 2014, 54, 61, 63, fig. 12.4-6.

11 CVArr $^{2}$ 2536.31-35.

12 I materiali provengono dalla US 140 "riempimento della fossa per la messa in opera della fogna" (scavi 1985, quadrati D-E, 3-4).

13 CVArr ${ }^{2}$ 270.11-12. La datazione è confortata anche dalla tipologia del piede (B 3.13), che rimanda a forme di età augustea e tiberiana, per cui cfr. Conspectus, 160, tav. 50.

14 RIC I ${ }^{2}$, 76.435: Roma, 7 a.C., AE, 10,98 g; asse $176^{\circ}$. D/ Testa a d. di Augusto, intorno [CAESA]R AVGVST PONT MAX TRIBVNIC [POT]. R/ Nel campo SC, intorno [M] MAECILIVS TVL[LVS] IIIVIR AAA[FF].

15 Gibbs 1976, 30-35.

16 Si deve a Bonnin 2015, 124-25, un chiarimento definitivo in merito alla terminologia latina e alla sua associazione a un determinato tipo di quadrante. 


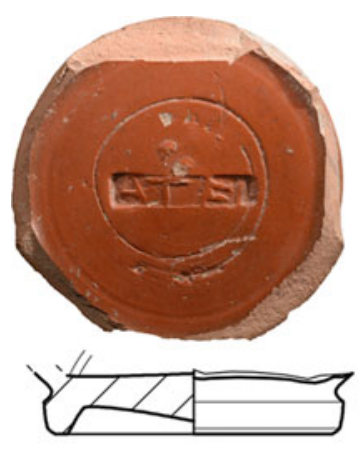

0

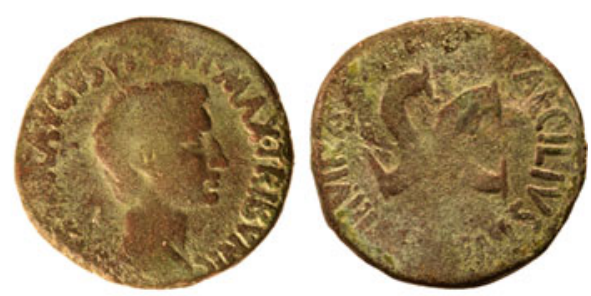

$5 \mathrm{~cm}$

Fig. 3. Materiali rinvenuti nella fossa di fondazione del condotto fognario. (E. Taccola.)

fonte antica più autorevole in merito, l'ideazione del quadrante conico si deve a Dionisodoro, ${ }^{17}$ anche se secondo studi recenti la paternità dell'invenzione è da attribuire ad Apollonio di Perga, matematico attivo ad Alessandria tra III e II sec. a.C. ${ }^{18}$

L'orologio è pressoché integro, a eccezione di abrasioni e lacune circoscritte che tuttavia non hanno pregiudicato né l'aspetto generale del manufatto, né, come vedremo oltre, lo studio gnomonico (Figs. 4-5). ${ }^{19}$

Una peculiarità che lo distingue all'interno del corpus degli orologi solari dell'antichità è relativa alle dimensioni estremamente ridotte dell'oggetto, che di seguito si riportano:

larghezza: $5.45 \mathrm{~cm}$

altezza: $5.22 \mathrm{~cm}$

profondità al piano dell'orizzonte (conservata): $2.86 \mathrm{~cm}$

profondità alla base: $2.46 \mathrm{~cm}$ sponde, $1.90 \mathrm{al}$ centro

larghezza lineare della superficie conica incisa (conservata): $4.35 \mathrm{~cm}$

altezza lineare della superficie conica incisa: $2.25 \mathrm{~cm}$

Il lato principale anteriore presenta una faccia obliqua, a formare un sottosquadro di $47^{\circ}, 55$ rispetto al piano orizzontale, entro la quale è ricavata la superficie conica. Su questa superficie sono segnate le tre curve di declinazione orizzontali, tra loro parallele, a indicare il solstizio d'inverno (in alto), gli equinozi (al centro) e il solstizio d'estate (in basso) e le undici linee orarie verticali (da sinistra a destra), di cui l'ottava interessata da una abrasione in basso e l'ultima interrotta da una frattura. La linea oraria centrale del mezzogiorno prosegue verso l'alto fino all'incavo dello gnomone, ricavato sulla faccia superiore, liscia e orizzontale. Il quadrante poggia su una basetta lavorata nello stesso blocchetto di avorio, in forma di piccolo podio di struttura trapezoidale, modanato sulla sola faccia anteriore e delimitato sui lati da sottili sponde aggettanti a profilo ondulato, di cui la destra lacunosa nella parte superiore. Il lato posteriore, verticale e liscio (con un'impressione ad anello

17 Benché Vitruvio non fornisca ulteriori dettagli, si ritiene che la personalità indicata sia il matematico Dionisodoro di Cauno, vissuto tra III e II secolo a.C.

18 La problematica è sintetizzata in Bonnin 2015, 124.

19 Il modello 3D interattivo dell'orologio solare di Pisa è disponibile al link https://visual.ariadneinfrastructure.eu/3d/209668e17d36f95e7e6a0d4ebf019fad. 


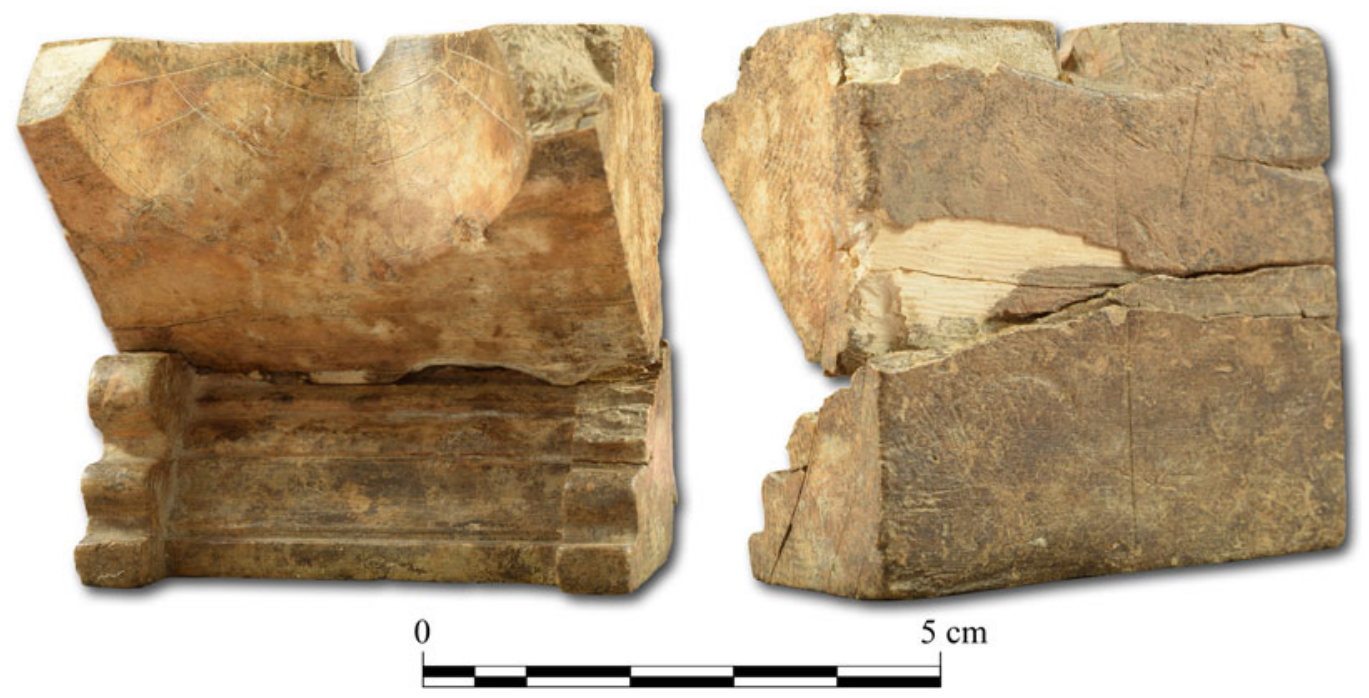

Fig. 4. Foto dell'orologio solare di piazza del Duomo. (E. Taccola.)
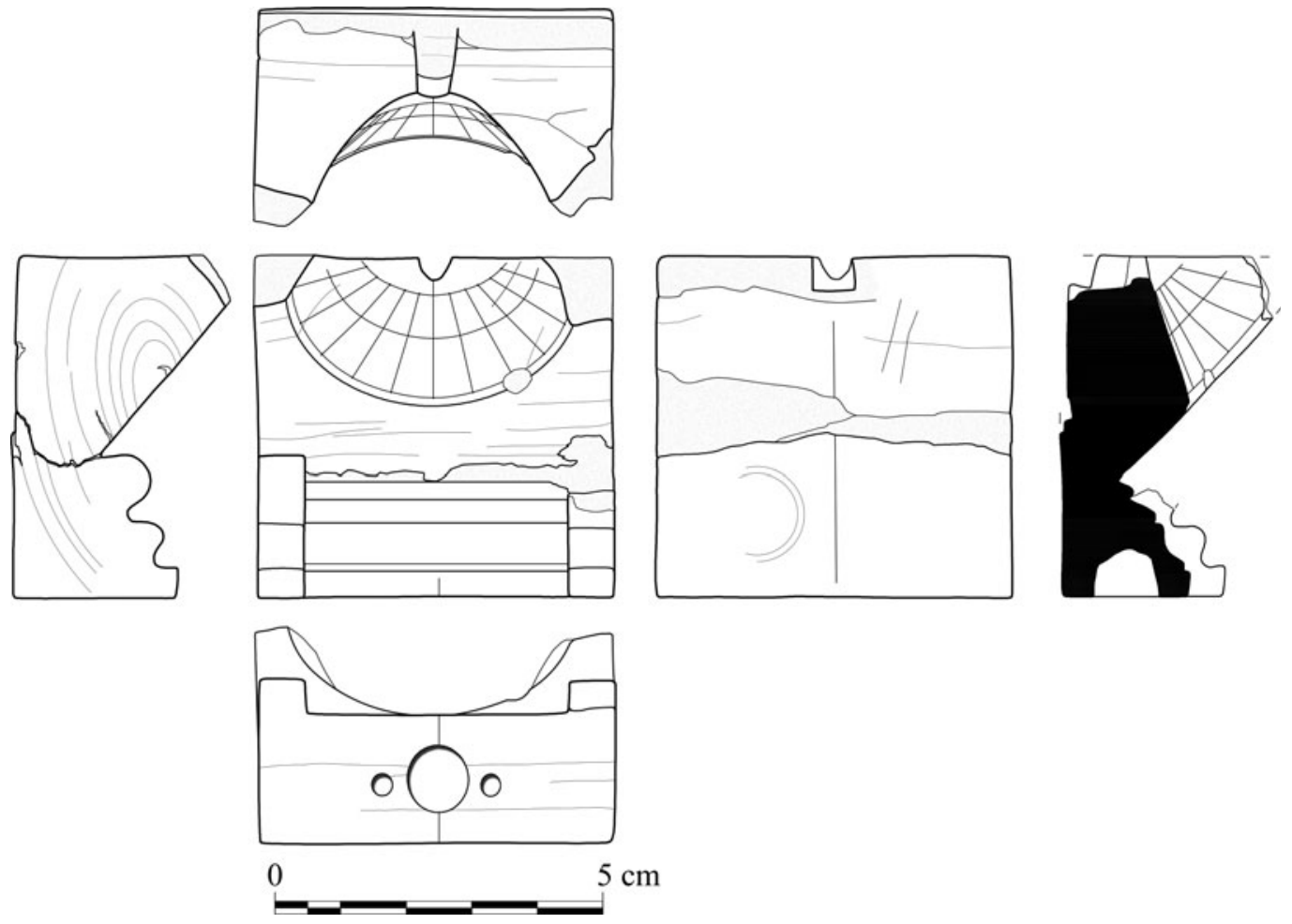

Fig. 5. Rilievo grafico dell'orologio solare di piazza del Duomo. (E. Taccola.)

appena percettibile di incerta natura), il piano di posa e l'estremità inferiore della basetta modanata sono percorsi da un solco rettilineo che segna l'asse centrale, in ideale continuità con la linea del mezzogiorno.

Sul piano di posa sono ricavati tre fori circolari allineati sul lato lungo: i due laterali hanno un diametro di 3,2 $\mathrm{mm}$ e una profondità di 5,1 $\mathrm{mm}$; quello al centro un diametro 

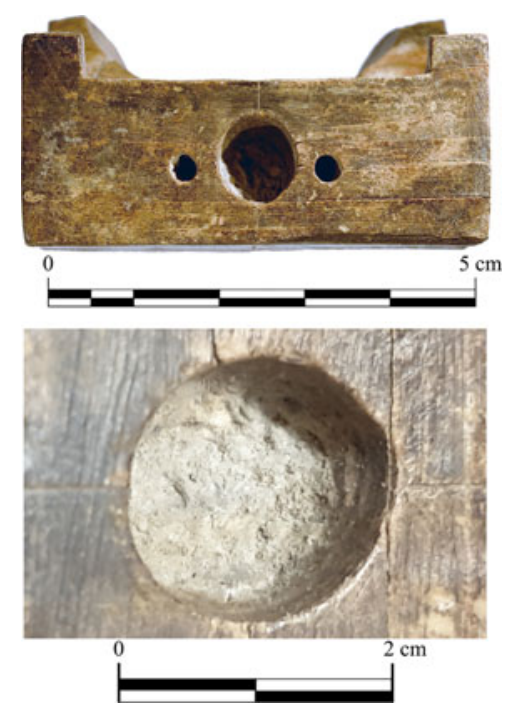

Fig. 6. Dettaglio della base. In basso è evidente il residuo metallico all'interno del foro centrale. (E. Taccola.)

di 9,7 mm e una profondità di 7,3 mm. L'interno della cavità centrale è foderato da una incrostazione metallica, riferibile quasi sicuramente a piombo (Fig. 6). Fori in questa posizione compaiono frequentemente sugli esemplari di dimensioni standard e indicano che tali oggetti erano fissati su un apposito supporto: ${ }^{20}$ la presenza dei fori e il residuo metallico suggeriscono che anche il quadrante miniaturistico è stato, almeno in una fase della sua "microstoria", saldamente assicurato a un sostegno.

L'eccellente stato di conservazione consente di apprezzare la sequenza di incisione della griglia oraria (Fig. 7): sulla superficie conica sono state inizialmente realizzate le curve di declinazione, che proseguono oltre la prima e l'ultima linea oraria; successivamente sugli archi dei solstizi sono stati visibilmente segnati i punti attraverso i quali far passare i solchi delle linee orarie. A giudicare dallo spessore medio delle incisioni, l'artigiano ha utilizzato uno strumento dotato di una punta di due decimi di millimetro.

Una ulteriore specificità del manufatto risiede nel materiale con cui è stato realizzato, l'avorio di elefante, ${ }^{21}$ che lo rende il secondo quadrante di questo tipo finora noto in letteratura, insieme a un esemplare conico di età tolemaica da Tanis, nel Delta egiziano, ricavato invece da un dente di ippopotamo. ${ }^{22}$ Quest'ultimo versa tuttavia in uno stato di conservazione decisamente precario a causa di un incendio che colpì l'edificio in cui è stato rinvenuto. Infatti, benché caratterizzato da un'iscrizione in greco e dall'indicazione delle curve diurne, oltre a quelle di declinazione e delle linee orarie, manca completamente della base e presenta una grande lacuna nella porzione destra della superficie conica incisa.

Tenendo conto che le dimensioni dell'esemplare egiziano sono molto simili, sia in larghezza che per le porzioni conservate della superficie conica incisa, ${ }^{23}$ e considerando più in generale la rassegna dei quadranti miniaturistici attualmente noti, illustrata nel paragrafo successivo, l'orologio di piazza del Duomo può essere annoverato tra i più piccoli, meglio realizzati e meglio conservati dell'antichità greco-romana.

20 A titolo esemplificativo segnaliamo un quadrante conico in marmo da residenza privata da Delo, datato al 100 a.C. ca.: oltre ad avere una struttura simile, con una base a due sponde, ma senza modanatura frontale, presenta sul piano di posa due fori quadrati con ancora aderente una fodera di piombo funzionale al fissaggio. L'oggetto è incluso nella raccolta degli orologi solari antichi (Ancient Sundials 2016), consultabile on-line nell'ambito della piattaforma di ricerca multidisciplinare Edition Topoi (http://repository.edition-topoi.org/collection/BSDP). Per la citazione del singolo esemplare nell'articolo utilizziamo l'abbreviazione Dialface ID seguita dal suo numero identificativo (nell'esempio citato, Dialface ID 654) con collegamento ipertestuale al database nella versione digitale del testo.

${ }^{21} \quad$ L'identificazione del tipo di avorio è stata operata dal dott. Y. Naime.

22 Dialface ID 317. Evans e Marée 2008, 1, n. 2. Vd. sotto, in particolare n. 45.

23. Evans e Marée 2008, 1. 


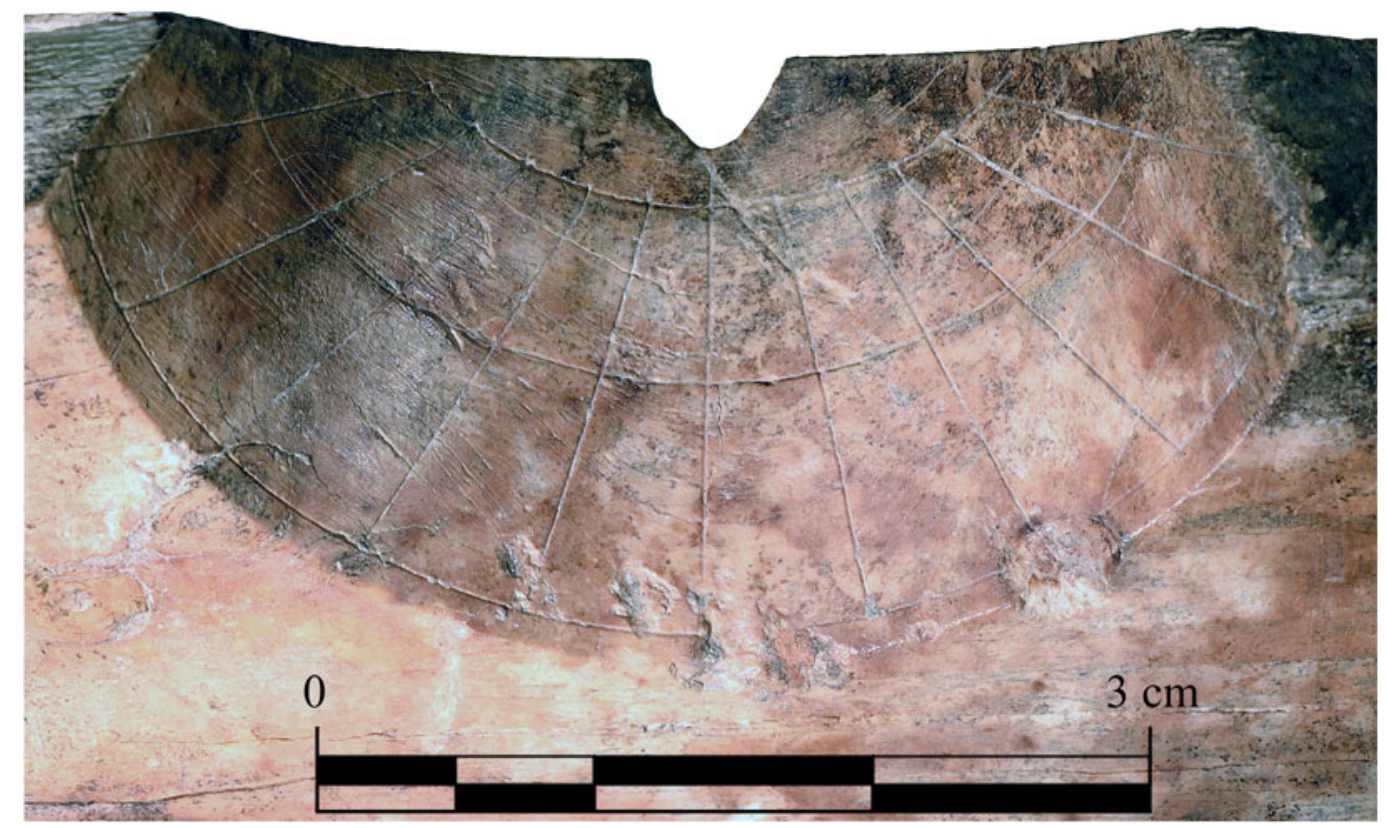

Fig. 7. Dettaglio della superficie conica incisa. (E. Taccola.)

\section{Datazione}

Riguardo alla datazione del quadrante pisano, come già anticipato, disponiamo di un terminus ante quem valido, compatibile con una rimodulazione urbanistica dell'area intrapresa a seguito della deduzione della Colonia Opsequens Iulia Pisana, tra il 41 e il 27 a.C., ${ }^{24}$ e in corso ancora allo scorcio del I sec. a.C.; più complicato è stabilire un terminus post quem, poiché il reperto è stato recuperato in deposizione secondaria, sebbene in un contesto certo, di natura residenziale.

Se quindi la giacitura stratigrafica del quadrante non contribuisce a determinare il limite cronologico alto, neppure i confronti stilistici per la resa della base modanata sono di conforto a tale scopo. Dalla consultazione del corpus degli orologi solari antichi emerge che un'impostazione simile del podio, con sponde laterali e modanatura anteriore più o meno schematica, è attestata in esemplari distribuiti tra la media età ellenistica e l'età imperiale. I paralleli più pertinenti per il profilo delle sponde si ritrovano su esemplari da Delo, Mykonos, Nafplion e Portogruaro: ${ }^{25}$ va detto però che in questi casi la modanatura anteriore è praticamente assente o appena accennata. Esempi simili per la modanatura anteriore ricorrono invece su basi prive delle sponde laterali: a titolo dimostrativo segnaliamo esemplari da Atene, Altino, Mileto, Paphos e Gerusalemme, databili tra la media età ellenistica e il I sec. d.C. ${ }^{26}$

Tenendo conto che il quadrante di tipo conico fece la sua comparsa in Grecia durante il III sec. a.C. (forse anche prima), ${ }^{27}$ possiamo supporre che tale modello sia stato recepito in

25 Rispettivamente: Dialface ID 654 (già citato a n. 20); Dialface ID 124; Dialface ID 125; Dialface ID 643; Dialface ID 480.

26 Rispettivamente: Dialface ID 144; Dialface ID 184; Dialface ID 312; Dialface ID 148; Dialface ID 432; Dialface ID 456.

$27 \quad$ Bonnin 2015, 124. 
Italia in tempi relativamente brevi, se è vero che a Roma gli strumenti per la misura del tempo furono introdotti già a partire dalla metà del III sec. a.C. ${ }^{28}$

Tra gli ultimi decenni del III e il primo quarto del II sec. a.C. il settore orientale di piazza del Duomo attraversò una fase di abbandono e graduale ripristino dell'area in seguito a eventi di natura alluvionale e antropica. ${ }^{29}$ Poiché il periodo di frequentazione precedente coincide cronologicamente con la comparsa in Grecia del quadrante conico, per la datazione dell'orologio miniaturistico da Pisa riteniamo più verosimile restringere la finestra temporale tra il secondo quarto del II sec. a.C., epoca in cui l'area della piazza del Duomo è convertita a quartiere residenziale, ${ }^{30}$ e lo scorcio del I sec. a.C., momento in cui viene realizzato il condotto fognario entro la cui fossa di fondazione è stato rinvenuto il quadrante. È il periodo che vede Roma consolidare definitivamente la sua egemonia del Mediterraneo dopo la definitiva sconfitta di Cartagine e la distruzione di Corinto (146 a.C.), che determinò l'annessione della Grecia alla provincia di Macedonia, e il 30 a.C., anno in cui anche l'Egitto, ultimo dei regni ellenistici, fu dichiarato provincia romana. A Pisa, questa fase storica corrisponde al pieno processo di romanizzazione della città, iniziato con la designazione a civitas foederata (ultimo trentennio del III sec. a.C.), ${ }^{31}$ proseguito con l'assegnazione del municipium (90 a.C. circa) $)^{32}$ e culminato con la deduzione della colonia durante il secondo triumvirato o nei primissimi anni del principato.

E.T.

\section{I quadranti miniaturistici nel corpus gnomonico antico}

L'orologio solare di Pisa non è un caso isolato, seppur i quadranti miniaturistici in avorio siano rari. Il fatto stesso che si tratti di un esemplare miniaturistico è di per sé notevole. Infatti, anche se non rappresenta una categoria tipologica a sé, in quanto gli strumenti che rientrano in questa denominazione hanno sempre l'aspetto di quadranti sferici o conici, cui si aggiunge un solo esemplare di tipo orizzontale, tali oggetti sono comunque caratterizzati da dimensioni estremamente ridotte. Tutti questi quadranti stanno nel palmo della mano di un adulto e non superano gli $11 \mathrm{~cm}$ di altezza. Il loro studio è delicato, perché spesso presentano tutte le caratteristiche di un quadrante "a grandezza naturale" (linee delle ore incise o dipinte, curve di declinazione, foro per l'alloggiamento di uno gnomone, decorazioni, ecc.), ma il più delle volte mancano di un'iscrizione o di un contesto di rinvenimento che consentano di conoscerne la funzione. Inoltre, il loro studio gnomonico è una vera sfida, viste le dimensioni. La maggiore concentrazione di oggetti di questa categoria si trova a Gerusalemme, vicino al Grande Tempio, con sei dei diciannove esemplari noti, compreso quello di Pisa. Tuttavia, di questi sei, due non sono che abbozzi di quadranti. Un altro gruppo più piccolo di quadranti miniaturistici è a Siracusa. I diciannove quadranti in miniatura ad oggi conosciuti sono distribuiti tra Turchia, Spagna, Italia, Grecia, Egitto e Israele (Tabella 1, Figs. 8-9). ${ }^{33}$

28

Bonnin 2015, 72. L'esemplare più antico finora rinvenuto su suolo italico è un quadrante di tipo sferico con iscrizione umbra in alfabeto etrusco da Mevania (Bevagna), datato tra la fine del III e gli inizi del II secolo a.C.: Bonnin 2015, 69-70 con riferimento a Filippetti 2000.

29 Taccola 2019, 24.

30 Cfr. n. 8.

31 Pasquinucci 1995, 311.

32 Harris 1971, 238, n. 3.

33 Escludiamo dal corpus l'orologio solare piano rinvenuto in Inghilterra nel sito di Fort Richborough nel 1926 e conservato a Dover (inv. 78303734). Infatti, si differenzia completamente 
Tabella 1.

Prospetto degli orologi solari miniaturistici finora noti

Turchia Efeso. Quadrante di

tipo sferico in

terracotta.

Dialface ID 694

Spagna Vilanova i la Geltrù. Quadrante di tipo

Iulia Libica.

Quadrante di tipo

piano orizzontale in

$\begin{array}{lc}\text { Dialface ID } 572 & \text { terracotta. Inedito } \\ \text { Siracusa. Quadrante di } & \text { Siracusa. Quadrante }\end{array}$

Italia Siracusa. Quadrante di Siracusa. Quadrante di Siracusa. Quadrante tipo conico in pietra tipo sferico in pietra di tipo sferico in

calcarea.

calcarea.

marmo bianco.

Pisa. Quadrante di

Dialface ID 478 Dialface ID $621 \quad$ Dialface ID 620

Grecia Delo. Quadrante di Delo. Quadrante di Rodi. Quadrante di tipo sferico in marmo tipo sferico in marmo tipo sferico in

bianco.

bianco.

marmo.

Dialface ID $653 \quad$ Inedito

Dialface ID 558

Egitto Naucratis. Quadrante Alessandria.

di tipo sferico in Quadrante in gesso.

calcare. Dialface ID 41 Dialface ID 448

Israele Gerusalemme.

Gerusalemme.

Quadrante di tipo

conico in pietra

calcarea.

Quadrante di tipo

sferico o conico in

pietra calcarea.

Tanis. Quadrante di

tipo conico in avorio.

Dialface ID 317

Gerusalemme.

Quadrante di tipo

sferico o conico in

Dialface ID 432

Macalister e Garrow

pietra calcarea.

Gerusalemme.

Quadrante di tipo

indeterminato in

pietra calcarea

Gerusalemme. Abbozzo

Gerusalemme. Abbozzo

di quadrante in

di quadrante in

materiale non

determinato. Dialface

determinato. Dialface

Duncan 1926,154-55

Adam 2002, 53

Adam 2002, 54 


\section{Emanuele Taccola et al.}

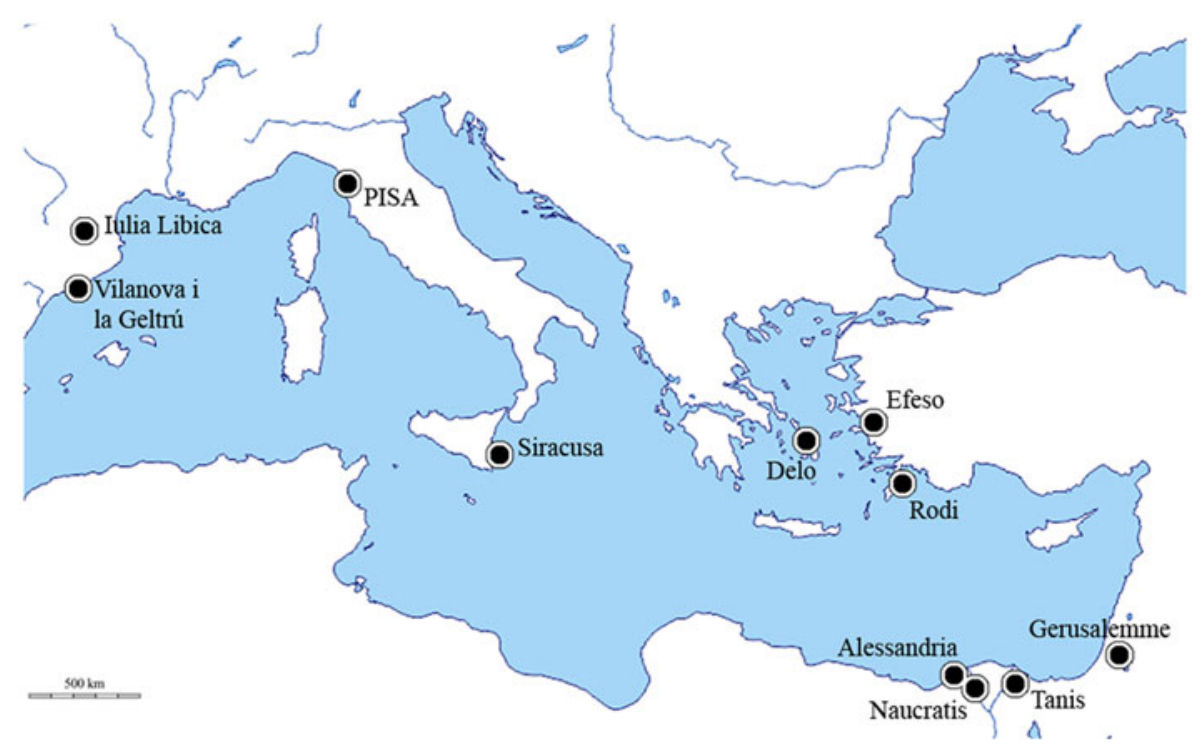

Fig. 8. Carta di distribuzione degli orologi solari miniaturistici. (E. Taccola.)
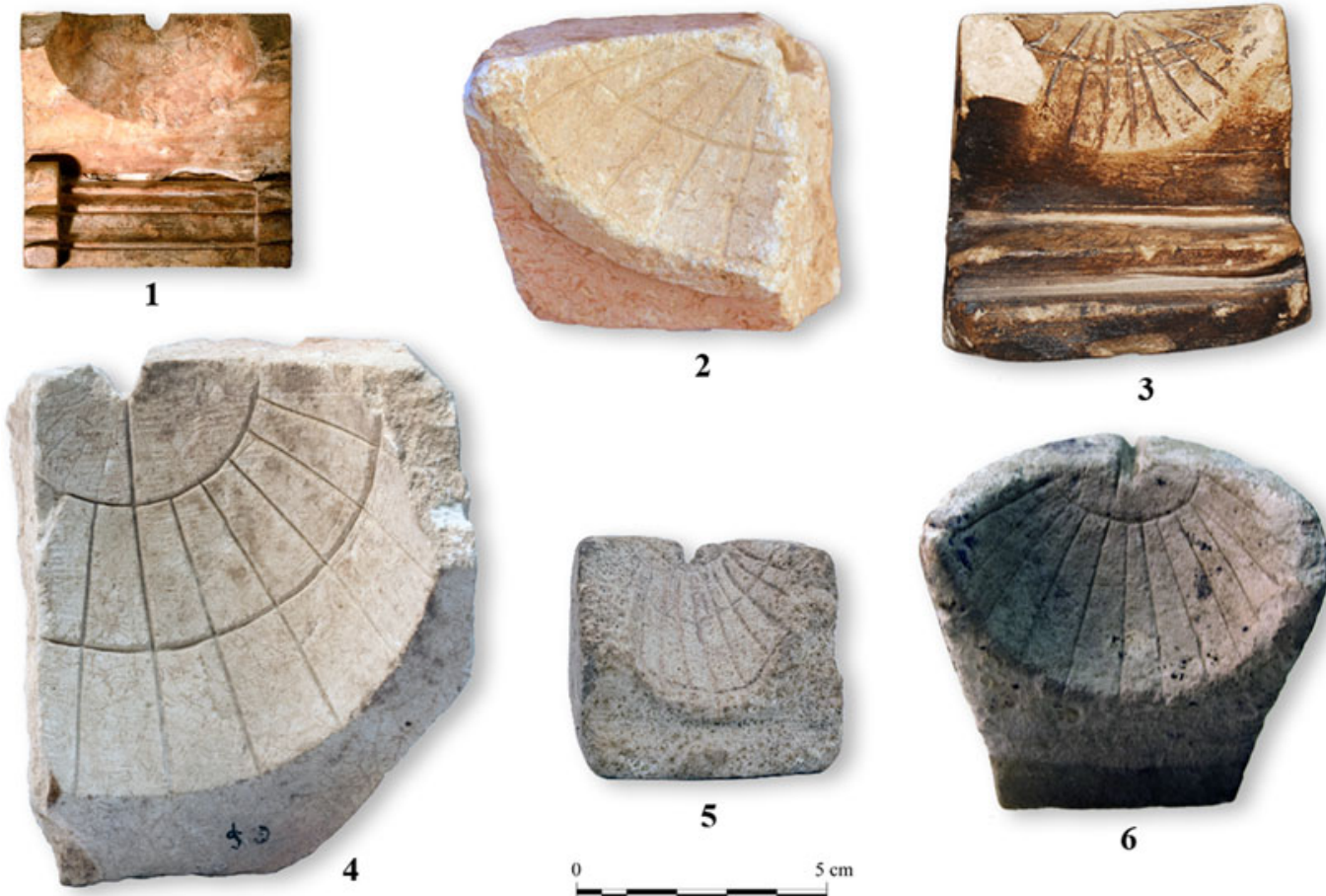

3
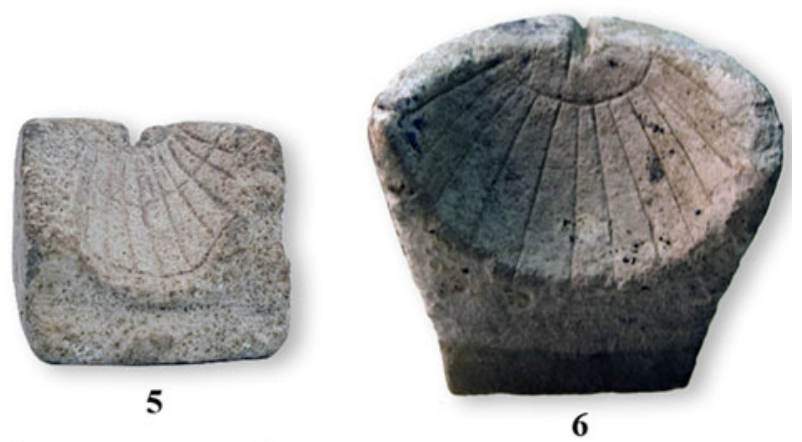

Fig. 9. Selezione di orologi solari miniaturistici scalati alla medesima dimensione. 1. Pisa; 2. Rodi, Dialface ID 558; 3. Gerusalemme, Dialface ID 432; 4. Siracusa, Dialface ID 620; 5. Siracusa, Dialface ID 478; 6. Naucrati, Dialface ID 41. (E. Taccola, immagini 2-6 courtesy Ancient Sundials 2016.)

per essere verticale e per essere particolarmente mal eseguito. Inoltre, si ritiene che sia l'unica meridiana romana rinvenuta sul suolo britannico, rendendola un esemplare unico da valutare con cautela. Cfr. Bonnin 2010, 36 e Taylor e Collingwood 1926, 239-43. 
In Turchia, l'unico quadrante miniaturistico conosciuto, in terracotta, è conservato a Efeso, da cui probabilmente proviene. Il museo locale lo data tra il IV e il I sec. a.C., ma una realizzazione tra I a.C. e I d.C. è più probabile. Lo strumento è di tipo sferico, senza curve di declinazione, con l'incavo per l'alloggiamento dello gnomone. ${ }^{34}$

Dalla Spagna proviene un quadrante di tipo sferico in marmo, scoperto nel 1991 a Vilanova ì la Geltrù, poco a sud di Barcellona, in un contesto di età repubblicana. ${ }^{35}$ Alto $8 \mathrm{~cm}$, conserva dieci linee orarie e la curva di declinazione equinoziale. Nel 2019 è stato rinvenuto un secondo esemplare miniaturistico negli scavi del foro di Iulia Libica, al confine pirenaico con la Francia. Realizzato in terracotta, è lungo 6,2 cm per $4 \mathrm{~cm}$ di larghezza e si data in età augustea. Tuttavia, più che un quadrante solare vero e proprio sembra piuttosto un modello, da attribuire a una produzione vernacolare e non di prestigio. ${ }^{36}$

In Italia esistevano finora tre quadranti miniaturistici, tutti provenienti dalla stessa città, Siracusa, conservati al Museo Archeologico Paolo Orsi. Il primo (inv. 35080) è un esemplare conico che presenta undici linee orarie e tre curve di declinazione. ${ }^{37}$ L'incavo di alloggiamento dello gnomone è ancora visibile. L'oggetto è imperfetto dal momento che è realizzato in una pietra calcarea che non consente una incisione precisa e sottile. Il secondo quadrante è di tipo sferico (inv. 97456). La pietra è dello stesso tipo del precedente e sembra far parte della stessa serie. ${ }^{38}$ Analogamente, l'esecuzione e la finitura sono particolarmente grossolane, assomigliando maggiormente a una imitazione di quadrante piuttosto che a un vero e proprio strumento funzionante. Il terzo quadrante siracusano (inv. 96141), di tipo sferico, è stato rinvenuto nella necropoli. ${ }^{39}$ Realizzato in marmo bianco, si data in età ellenistica; la parte conservata misura $11 \mathrm{~cm}$ di altezza, mentre la larghezza originaria doveva raggiungere i $20 \mathrm{~cm}$.

In Grecia, due quadranti miniaturistici, entrambi inediti, provengono da Delo, mentre un terzo viene da Rodi. Il primo, alto $8 \mathrm{~cm}$, è conservato nei depositi del museo di Delo (inv. B 18130). ${ }^{40}$ Di tipo sferico, è realizzato in marmo bianco e conserva tre curve di declinazione e la linea meridiana dipinte in rosso. Le altre linee orarie, che dovevano essere tracciate in nero, sono scomparse. La forma in generale richiama quella degli strumenti di dimensioni standard di Cos e Rodi. Il secondo strumento, fratturato in due parti, non supera i $10 \mathrm{~cm}$ di altezza. ${ }^{41}$ Di tipo sferico, comprende dodici settori orari e tre curve di declinazione. La curva equinoziale è molto ravvicinata a quella del solstizio d'inverno. Lo gnomone era costituito da un'asticella cilindrica di metallo fissata al quadrante con un gancio, di cui rimane la traccia. Il terzo è segnalato nella raccolta Topoi come conservato al Museo Archeologico di Rodi (inv. APX 1113). ${ }^{42}$ Si tratta di un frammento di quadrante di tipo sferico realizzato in pietra, forse marmo.

\footnotetext{
34 Dialface ID 694, Ephesus Archaeological Museum, Selçuk (senza inv.).

35 Dialface ID 572, Museu Víctor Balaguer, Vilanova i la Geltrú (senza inv.). Gumà i Marquès 1992.

36 Carreras e Farré in stampa.

37 Dialface ID 478.

38 Dialface ID 621.

39 Lo Sardo 2005, 249 (Dialface ID 620).

40 Dialface ID 653.

${ }_{41}$ Il quadrante è tuttora inedito. È stato scoperto prima del 2009 e mi è stato segnalato da J.-S. Gros, allora membro dell'EFA.

42 Dialface ID 558.
} 
In Egitto, sono stati rinvenuti tre orologi solari della stessa tipologia. Il primo a Naucrati nel 1883, nel santuario di Apollo, conservato al British Museum (inv. 1909 2-16 10). ${ }^{43}$ Realizzato in calcare, misura $6,5 \mathrm{~cm}$ in altezza e presenta dodici settori orari, ma nessuna curva di declinazione. Al di sotto si intravede la traccia di alcune lettere, sfortunatamente non decifrabili nel loro insieme. Il secondo proviene da Alessandria ed è stato portato alla luce all'inizio del XX sec. a Ibrahimiya, un'area costiera situata nei pressi della porta principale orientale della città (Kanopike Pyle). ${ }^{44}$ L'oggetto, conservato nel Museo greco-romano di Alessandria (inv. 15735), è descritto da Locher come modellato in gesso, caratteristica che lo rende un unicum, sebbene non si conoscano altre informazioni tecniche o storiche. Il terzo quadrante proviene da Tanis ed è stato scoperto nel 1883 da W. M. Flinders Petrie nei resti di una residenza privata di età tolemaica. Attualmente è custodito presso il Department of Ancient Egypt and Sudan del British Museum (inv. EA 68475). Come già menzionato, è un quadrante conico realizzato in avorio di ippopotamo, conservato per un'altezza di $3 \mathrm{~cm}$ su un'originaria dimensione di $5 \mathrm{~cm}$ e corredato da

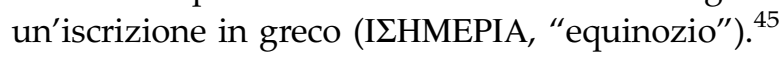

In Israele sono stati trovati non meno di sei orologi solari in miniatura, tra cui due appena abbozzati, tutti a Gerusalemme. Il primo è stato scoperto nel 1925 ai piedi dell'angolo sud-ovest del monte del tempio. ${ }^{46}$ In calcare, misura $6 \mathrm{~cm}$ di altezza e presenta 11 linee orarie e una curva di declinazione che sembra essere quella degli equinozi. La faccia posteriore è decorata con una menorah e conserva due mortase. La presenza della menorah permette di datare l'oggetto all'inizio del primo sec. d.C. L'incisione è imprecisa a tal punto che era impossibile leggervi l'ora. Il secondo orologio è stato scoperto tra il 1925 e il 1926 sull'Ophel. ${ }^{47}$ In calcare, misura $9 \mathrm{~cm}$ in altezza e presenta tutte le linee orarie e tre curve di declinazione. Il terzo è stato rinvenuto nel quartiere ebraico di Gerusalemme. ${ }^{48}$ In calcare, alto $11 \mathrm{~cm}$, conserva le linee orarie ed è decorato sulla faccia laterale destra da una serie di rosette incise. Anche il quarto orologio è stato rinvenuto nel quartiere ebraico ed è realizzato in calcare, misurando $5 \mathrm{~cm}$ in altezza. ${ }^{49} \mathrm{La}$ forma, tuttavia, è estremamente insolita dal momento che si tratta di un cubo svuotato, inizialmente utilizzato per una funzione diversa e non determinabile, che venne poi tagliato obliquamente. Si distingue un incocco sul bordo superiore e varie linee incise a mano, in modo impreciso, che si estendono a raggio a partire dall'incocco in direzione dei bordi. Si tratta di un esercizio, di un tentativo? Questo esemplare si avvicina ai due ultimi da Gerusalemme che costituiscono degli abbozzi di quadrante piuttosto che strumenti

43 Gibbs 1976, 155; Petrie 1886, 16, tav. 18.6 (Dialface ID 41).

$44 \quad$ Locher 1993 (Dialface ID 448).

45 Evans e Marrée 2008 (Dialface ID 317), cfr. nn. 22 e 56. L'iscrizione si trova sul piano frontale obliquo, in basso a destra. Orientato correttamente, il piano in sottosquadro del quadrante restava illuminato per tutto il giorno a partire dall'equinozio di autunno e fino all'equinozio di primavera, rimanendo in ombra per tutta l'estate fino al successivo equinozio. Quindi l'illuminazione del piano in sottosquadro indicava l'equinozio, segnalato in quella posizione proprio dall'iscrizione I $\Sigma$ HMEPIA.

46 Dialface ID 432, Hecht Museum, Università di Haifa (inv. 1986-243). Adam 2002, 55.

47 Dialface ID 465, attualmente scomparso. Macalister e Garrow Duncan 1926, 154-55.

48 Dialface ID 456, Wohl Archaeological Museum, Gerusalemme (inv. 1982-1020). Adam 2002, 53.

49 Adam 2002, 54. 
realmente funzionanti. Sono stati rinvenuti nel quartiere ebraico durante gli scavi di N. Avigad e H. Geva. ${ }^{50}$ Il materiale è sconosciuto, entrambi gli orologi hanno la stessa forma, molto rudimentale, e misurano $6 \mathrm{~cm}$ in altezza. Sono stati realizzati con gli scarti di un laboratorio di tornitura, le tracce del tornio sono ancora visibili sulla superficie esterna. La faccia superiore del cilindro è stata scavata per dare una forma sferica/concava alla superficie analemmatica. Un incocco è stato inciso sul bordo superiore per consentire di alloggiarvi lo gnomone, mentre alcune linee orarie sono state incise a mano e senza alcuna precisione, dall'incocco fino al bordo inferiore.

J.B.

\section{Studio gnomonico del quadrante di Pisa}

Come indicato nella sezione precedente, cercare di estrarre parametri gnomonici affidabili dal quadrante di Pisa è praticamente impossibile. Infatti, su un quadrante "classico", cioè di dimensioni normali (tra 30 e $50 \mathrm{~cm}$ di larghezza ad esempio), un errore di misura di vari millimetri può talvolta portare a un risultato distorto di alcuni gradi. Che dire allora del quadrante di Pisa, che misura appena 5,22 cm di altezza e la cui lunghezza totale della linea meridiana è di soli $1,53 \mathrm{~cm}$ ? In questo caso, gli errori di misurazione non sono più ipotetici ma certi.

Tuttavia, per sapere se uno schema matematico abbia presieduto alla costruzione di questa meridiana, è possibile effettuare alcune misurazioni e applicare le formule che ho elaborato per la determinazione dei parametri gnomonici di una meridiana antica, ${ }^{51}$ in particolare la latitudine del luogo per il quale teoricamente la meridiana doveva funzionare. Perciò, assumiamo come ipotesi di partenza che $\varepsilon$ (obliquità dell'eclittica) $=24^{\circ}$; lo scarto rispetto al reale non ha alcun effetto evidente sul risultato finale.

Sulla linea del "mezzogiorno", vale a dire la $6^{\mathrm{a}}$ ora disuguale, è possibile misurare le seguenti distanze (Fig. 10): ${ }^{52}$

Piede dello gnomone - equinoziale $(P Q)=8.6 \mathrm{~mm}$

Equinozio - solstizio d'estate $(\mathrm{QE})=8.9 \mathrm{~mm}$

Equinozio - solstizio d'inverno $(\mathrm{QI})=6.4 \mathrm{~mm}$

da cui distanza solstizio d'inverno - solstizio d'estate (IE) $=8.9+6.4=15.3 \mathrm{~mm}$

Angolo $\theta$ (angolo tra lo gnomone orizzontale o un piano orizzontale equivalente e la linea di massima inclinazione, mezzogiorno): $71^{\circ}, 58$

Dalla formula $\tan \omega=(\mathrm{QE}-\mathrm{QI}) /$ IE $\tan \varepsilon$, si ottiene l'angolo $\omega$ (angolo tra l'asse centrale del cono e una qualsiasi generatrice $)=20^{\circ}, 15$

Ciò determina una prima ricerca della latitudine, a partire dalla formula $\theta=\omega+\varphi$

$\left(\varphi=71^{\circ}, 58-20^{\circ}, 15\right)$, quindi $\varphi=51^{\circ}, 43\left(\right.$ o $\left.51^{\circ} 26^{\prime}\right)$

50 Dialface ID 748; Dialface ID 749. Wohl Archaeological Museum, Gerusalemme (senza inv.). Adam 2002, 54.

51 Savoie 2020, 48-50 specificamente per i quadranti conici.

52 Le misure sono quelle fornite da E. Taccola, calcolate sul rilievo fotogrammetrico 3D del quadrante (vd. n. 19). 


\section{Emanuele Taccola et al.}

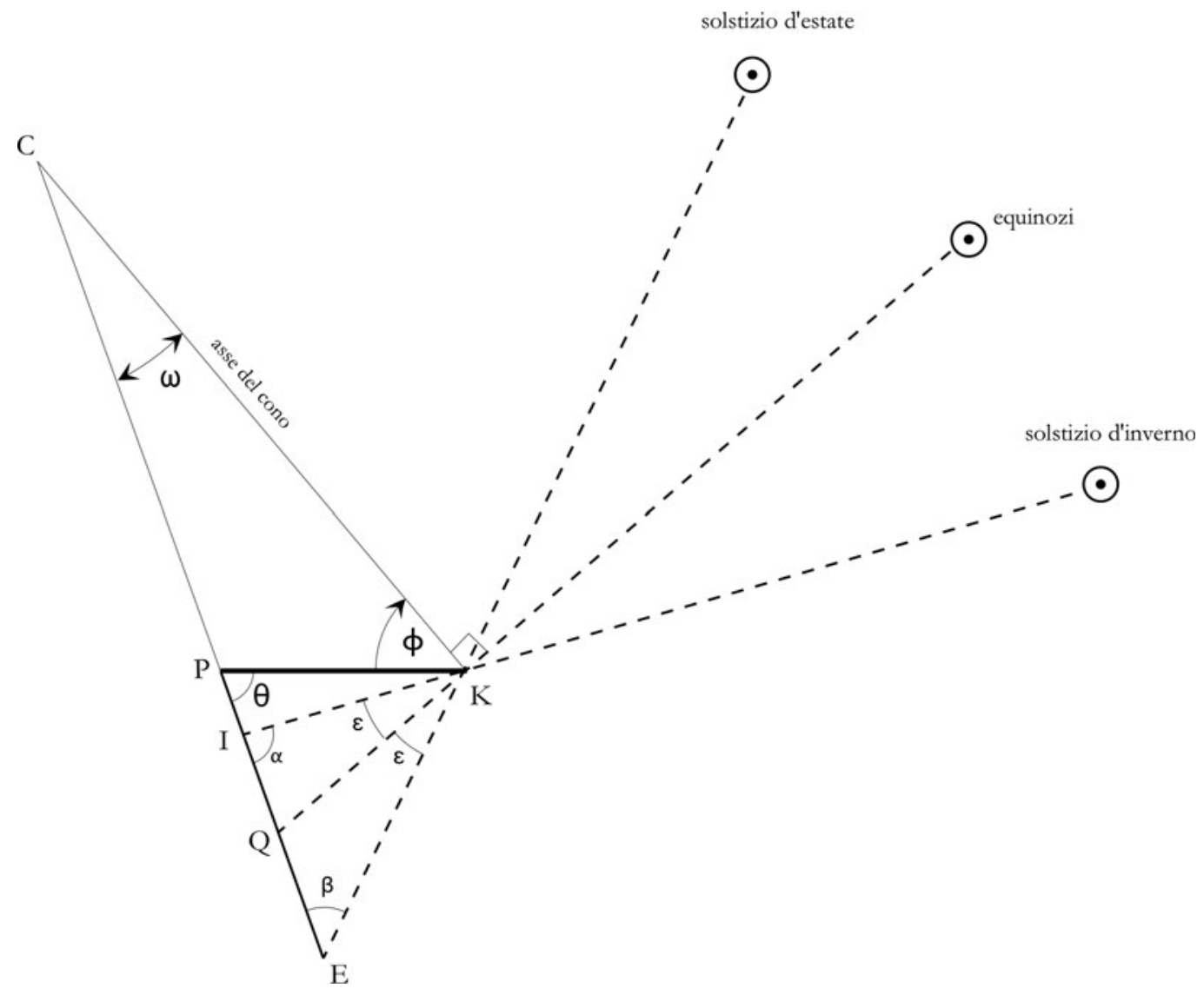

Fig. 10. Schema matematico del quadrante conico di Pisa. (D. Savoie.)

Un altro metodo indiretto consente di trovare la latitudine misurando le distanze QE (o QI) e PQ, dalle seguenti formule:

$\mathrm{QK}=\mathrm{QE} \cos (\omega+\varepsilon) / \sin \varepsilon$

$\mathrm{QK}=8.9 \mathrm{~mm} \times \cos 44.15 /$ seno 24

$\mathrm{QK}=15.7 \mathrm{~mm}$

oppure

$\mathrm{QK}=\mathrm{QI} \cos (\omega-\varepsilon) / \sin \varepsilon$

$\mathrm{QK}=6.4 \mathrm{~mm} \times \cos -3.85 / \sin 24$

$\mathrm{QK}=15.7 \mathrm{~mm}$

Questo permette di dedurre la lunghezza dello gnomone:

$\mathrm{PK}^{2}=\mathrm{PQ}^{2}+\mathrm{QK}^{2}-2 \mathrm{PQ} \times \mathrm{QK} \sin \omega$

$\mathrm{PK}^{2}=8.6^{2}+15.7^{2}-(17.2 \times(15.7 \times \sin 20.15))$

$\mathrm{PK}=15.1 \mathrm{~mm}$

Da questa lunghezza PK è possibile quindi ricavare la latitudine di costruzione del quadrante sulla base della formula $\cos \varphi=(\mathrm{PQ} / \mathrm{PK}) \cos \omega$ 
$\cos \varphi=(8.6 / 15.1) \times \cos 20.15$

allora $\varphi=57^{\circ}, 68\left(\right.$ o $\left.57^{\circ} 41^{\prime}\right)$

In ogni caso, si arriva a una latitudine molto lontana da Pisa che è $43^{\circ} 43^{\prime} \mathrm{N}$ (Fig. 11). È facile dimostrare che, sbagliando ad esempio di $1.4 \mathrm{~mm}$ sulla sola posizione della curva dell'estate, si ottiene una latitudine vicina a $46^{\circ}$; altrimenti un errore di misura molto piccolo si traduce in più di $11^{\circ}$ nel risultato finale.

Esiste un ultimo metodo che tiene conto della lunghezza totale dell'arco diurno o di parti dell'arco diurno estivo o invernale, metodo tuttavia molto sensibile agli errori di misura. Con l'arco estivo, si ottiene una latitudine media di $41^{\circ}$, questa volta più vicina a quella di Pisa. Con l'arco invernale si ottiene invece una latitudine media di $51^{\circ}, 5$. Da notare che, con questo arco invernale, la distanza tra due linee orarie è dell'ordine di $2 \mathrm{~mm}$ e che è estremamente complicato garantire il decimo di millimetro, essendo di per sé difficile apprezzare lo spessore della linea di incisione. Tuttavia, come spesso accade in gnomonica, una meridiana trovata in un luogo non significa che sia stata disegnata per quel luogo: il quadrante potrebbe essere stato progettato per funzionare a Roma (latitudine molto vicina a $41^{\circ}$ ) ed essere stato successivamente spostato a Pisa. Date le ridotte dimensioni del quadrante, il suo proprietario non sarebbe stato comunque in grado di evidenziare o apprezzare l'incidenza di uno spostamento di $2^{\circ}$ di latitudine sulla lettura dell'ora.

In definitiva, è difficile estrarre parametri realmente utilizzabili da tale quadrante. $\mathrm{Al}$ massimo si può dire che uno schema matematico ha presieduto alla sua realizzazione, ma senza alcuna accuratezza. Va anche ricordato che, per essere funzionante, questo strumento doveva essere allineato precisamente con il meridiano locale (direzione nord-sud): un errore di pochi gradi nell'orientamento sarebbe stato catastrofico. Sebbene ci sia un foro di tenuta sotto la base, che tende a dimostrare che l'orologio era posizionato correttamente, è più che probabile che non sia stato utilizzato per indicare con precisione l'ora. Forse la sesta ora, con un po' più di accuratezza. Si pone qui, come in oggetti simili, la questione dell'uso effettivo di questi manufatti sorprendenti, preziose curiosità come alcuni esemplari portatili molto piccoli del XVI e XVII sec., tra cui gli orologi solari ad anello. La funzione di prestigio legata all'utilizzo dell'avorio di elefante è senza dubbio quella primaria di questo minuscolo quadrante conico, in cui l'esattezza della griglia non era certo una priorità.

D.S.

\section{Note sugli aspetti sociali degli orologi solari in miniatura}

Gli orologi solari in miniatura costituiscono, nel Mediterraneo greco-romano, un gruppo eterogeneo ed estremamente peculiare. Le fonti antiche non sembrano menzionarli per questa loro specificità e i contesti archeologici di rinvenimento offrono spesso informazioni insufficienti. Le proposte che presento partono da due presupposti: 1) gli orologi in miniatura non avevano come scopo principale la misurazione del tempo, essi si inserivano ovviamente in questo orizzonte concettuale, ma il loro valore era piuttosto simbolico, come ben chiarito nella sezione precedente; 2) pur in un insieme ristretto di testimonianze non dobbiamo pensare che questi orologi abbiano avuto uno stesso (simbolico) scopo o che siano da riportare a contesti sociali affini. 

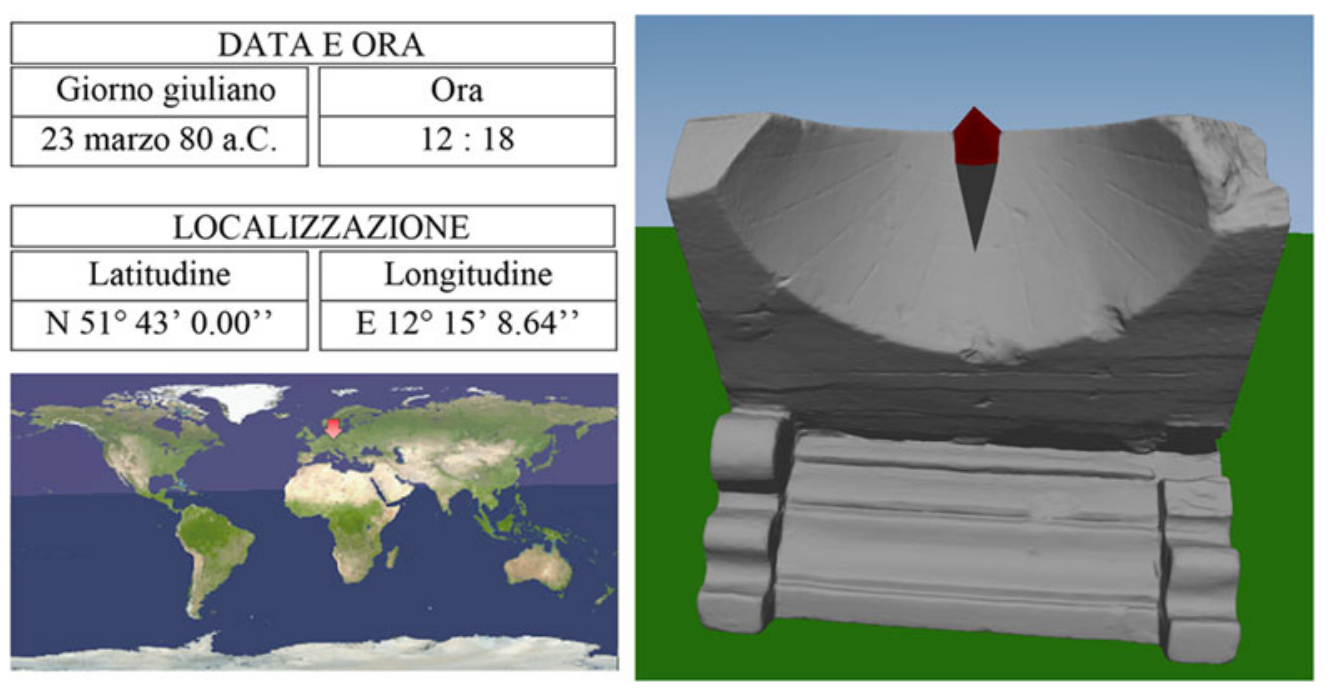

Fig. 11. Simulazione virtuale dell'ombra proiettata dallo gnomone sulla superficie conica alle ore 12:18 del 23 marzo dell'80 a.C., visualizzata sull'applicazione Stellarium (http://stellarium.org/). La data corrisponde all'equinozio di primavera del I secolo a.C. nel calendario giuliano (Savoie 1989, 3) alla latitudine di 51, 43. (E. Taccola).

A proposito della capacità di misurazione del tempo di questi orologi, vi sono alcuni problemi di una duplice natura, inerenti e accidentali. Tra i primi si annoverano le dimensioni minute che rendono la realizzazione della griglia oraria difficile e facilmente soggetta a imprecisioni. Da un punto di vista di uso ci si può chiedere inoltre quanto lo strumento fosse di facile lettura, considerata la necessità di avvicinarsi a causa delle dimensioni dell'oggetto e il conseguente rischio di coprire l'ombra. Tra i secondi, gli accidentali, bisogna tener conto della realizzazione di scarsa qualità che caratterizza un certo numero di questi orologi, rendendone alcuni completamente inutili ai fini della misurazione del tempo (vd. sopra).

Vi è poi una serie di paradossi: questi minuscoli orologi erano maneggevoli e facilmente spostabili, ma non hanno niente a che fare con quelli così detti portatili che potevano essere adattati - pur con molti limiti - alle coordinate del luogo in cui ci si trovava. ${ }^{53}$ Alle coordinate per cui erano stati creati, perché funzionassero era inoltre necessario che fossero orientati in maniera rigorosa, preoccupazione che sembra presente nel caso di Pisa, ma non in altri. D'altra parte, la maneggevolezza è necessaria per mitigare gli effetti concreti di un ulteriore aspetto paradossale: gli orologi solari del tipo di cui quello di Pisa costituisce un esempio miniaturistico sono concepiti per essere posti stabilmente all'aperto, ma se si fosse lasciato, come avviene di solito per gli orologi in pietra, un orologio di avorio continuamente esposto agli agenti atmosferici (in particolare sole, pioggia e sbalzi di temperatura) questo si sarebbe rapidamente danneggiato. ${ }^{54}$ Fermo restando che il paradosso è

53 Talbert 2017; Savoie 2020. Bonnin 2015, 120, 126-27. L'osservazione che propongo, riferita al tipo

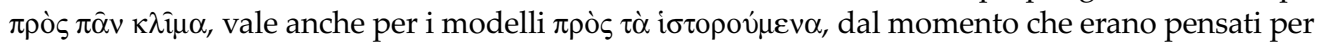
essere spostati. Mutatis mutandis, al solo fine di evidenziare lo scarto concettuale rispetto alla tipologia (stazionario vs. mobile), potremmo paragonare l'orologio solare pisano a un orologio a pendola minuscolo, mentre quelli portatili sarebbero piuttosto degli orologi a cipolla.

54 La delicatezza dell'avorio era ben presente anche in antichità, come mostrano ad esempio le cure per la statua fidiaca di Atena Parthenos, cfr. Lapatin 2001, 85-86. Diverso il caso degli orologi 
connaturato in questi oggetti, essi erano d'altra parte immediatamente riconoscibili e rimandavano a una sfera di conoscenze e significati ben precisa. Anche dal punto di vista della misurazione del tempo non erano completamente inutili, offrendo un'idea dello scorrere del tempo, ed erano perfettamente funzionanti, se orientati correttamente, ogni giorno a mezzogiorno e durante gli equinozi (supponendo che la griglia oraria fosse almeno decente) ${ }^{55}$ Sono tentato di leggere il caso di Tanis come prova del fatto che i limiti scientifici del mezzo erano ben presenti a chi lo realizzava. La scientificità veniva sottolineata puntando su un aspetto macroscopico di questo strumento microscopico: il passaggio equinoziale indicato con l'iscrizione I $\Sigma$ HMEPIA, che segnalava la parte che non sarebbe stata illuminata tra l'equinozio di primavera e quello d'autunno, materializzava una conoscenza scientifica che offriva un'informazione - per quanto superflua chiara ed immediata sul periodo dell'anno in cui ci si trovava. Inoltre tale indicazione avrebbe potuto favorire la discussione attorno all'oggetto e alla sfera di conoscenze cui rimanda, all'interno del possibile contesto di fruizione che verrà menzionato più avanti. ${ }^{56}$

Se lasciamo da parte i limiti funzionali da esse generati, le dimensioni minute, a volte minuscole, sono di per sé importanti e rimandano a una retorica e a un'estetica ben precisa. Ciò vale in particolar modo per i casi di Pisa e Tanis che non solo sono da annoverare tra gli orologi solari più piccoli ${ }^{57}$, ma anche tra quelli più rifiniti e dettagliati. Evidentemente il materiale scelto, l'avorio, costituisce un aspetto strettamente collegato, sia per le possibilità che offre nella lavorazione, sia per i maggiori costi, indice di un progetto più ambizioso. Se prendiamo nello specifico il nuovo caso pisano, osserviamo che si tratta non solo di una miniatura, ma di un oggetto miniaturizzato - uso l'espressione per sottolineare lo scarto che vi è con la quasi totalità degli altri orologi miniatura conservati - che in poco più di $5 \mathrm{~cm}$ di altezza presenta la base modanata e il quadrante di una meridiana regolare. Un lavoro che si avvicina ai mirabilia di Callicrates e Myrmecides di cui parla Plinio, anch'essi in avorio, particolare non casuale (HN 7.85):

\footnotetext{
"Callicrates realizzò delle formiche di avorio e altri animali tanto piccoli che gli altri non ne potevano vedere le parti che li costituivano. Myrmecides si distinse nel medesimo ambito, avendo realizzato, dello stesso materiale, una quadriga che poteva essere coperta da una mosca ad ali aperte, e una nave, che poteva essere nascosta dalle ali di un'apetta" (trad.: autore).
}

portatili che venivano esposti solo occasionalmente e che infatti vennero non raramente realizzati in avorio (gli esempi sono di età moderna).

55 Si vedano le utili considerazioni a proposito degli orologi solari in generale di Jones 2020, spec. $138-43$ e D. Savoie nella sezione precedente.

56 Evans e Marée 2008, 3-4, vd. nn. 22 e 45. In quest'ottica si noti anche la presenza degli archi diurni zodiacali, assenti nel modello pisano.

57 Le dimensioni contano, tanto più quanto più piccolo è l'oggetto. Credo sia necessario distinguere sia in base alle dimensioni assolute sia in base a quelle delle singole parti. L'orologio di Pisa che misura poco più di $5 \mathrm{~cm}$ di altezza va confrontato ad esempio con quello dalla necropoli di Siracusa in cui la sola parte conica aveva un'altezza di quasi $8 \mathrm{~cm}$ e integro raggiungeva forse i $20 \mathrm{~cm}$ (vd. n. 39) con la stessa cautela con cui lo si deve confrontare con quello di Rodi (vd. n. 42), di altezza paragonabile, ca. $6 \mathrm{~cm}$ (ma integro era largo probabilmente quasi 12!), che era costituito solo dalla parte conica, praticamente senza base. 
Nel campo del reale, o quantomeno di quanto è conservato, si può pensare al capolavoro di miniaturizzazione rappresentato dalle tabulae Iliacae. ${ }^{58}$

Oltre alle dimensioni, nei casi di Pisa e Tanis anche il materiale è significativo, sia socialmente sia culturalmente. ${ }^{59}$ Dal punto di vista sociale l'avorio si distingue infatti per i costi elevati e quindi il prestigio che ne derivava, dal punto di vista culturale, cosa che vale per Pisa e meno per Tanis, esso rimanda all'Egitto e costituisce un riferimento carico di significati per quanto riguarda astronomia e misurazione del tempo. Uno tra gli esempi più colossali è costituito dalla meridiana di Augusto a Roma, il cui gnomone era un obelisco egiziano. Il legame è reso esplicito dall'iscrizione alla base (CIL VI 762), 5-7: Aegypto in potestatem I populi Romani redacta | Soli donum dedit. ${ }^{60}$ Estremizzando, se si considera prioritario l'aspetto sociale si potrà pensare a un proprietario alla Trimalcione, che voleva veicolare un'immagine di sé da ricco intenditore. Se invece si pone l'accento sul significato culturale, si immaginerà un proprietario alla Mecenate, che, da colto intenditore, avrebbe potuto richiamare, ad esempio, la sapienza egiziana.

Per poter identificare i diversi contesti sociali, intesi come occasioni, in cui collocare questi oggetti, sarebbe utile poter determinare quelli archeologici. Quando ciò avviene, mi pare che le ipotesi si restringano significativamente. ${ }^{61}$ A Siracusa un esemplare è stato trovato in una necropoli e la presenza di un orologio, come metafora dell'inevitabilità del tempo, ben si inserisce in ambito funerario. ${ }^{62}$ A Naucrati, W. Flinders Petrie interpretava la meridiana trovata negli scavi del tempio di Apollo come un dono al dio, una spiegazione plausibile e convincente. ${ }^{63}$ In entrambi i casi il valore simbolico era prioritario, la concreta misurazione del tempo secondaria. Gli orologi di Tanis e di Vilanova vengono invece da abitazioni e anche quello di Pisa sembra da riferire a zona residenziale. ${ }^{64}$ In base a quanto detto finora vengono in mente due ipotesi, che non esauriscono tutte le possibilità, ma, nel campo delle congetture, mi paiono le più promettenti. La prima possibilità è che si tratti di meridiane giocattolo. I paralleli non mancano sia come tipologia di oggetti, piccoli vasi, tavoli, lucerne ecc., sia come materiali, oltre all'argento anche l'avorio era utilizzato. ${ }^{65}$ Tra i doni per Era si trovava ad esempio un piccolo giaciglio decorato con avorio, un giocattolo che era appartenuto a Ippodamia, ${ }^{66}$ ma il contesto - votivo - può aver pesato sulla scelta del materiale, vale a

58 Per le tabulae si veda Petrain 2014 e soprattutto Squire 2011, con ampia teorizzazione della questione dimensionale, 247-73. In generale sulla miniatura Stewart 1993.

59 Sull'avorio e la sua percezione/valore in età primo imperiale vd. ora Heinemann 2021.

60 Sul monumento vd. ora la sintesi di Bonnin 2020.

61 Lascio da parte gli esemplari di Gerusalemme, che per qualità e quantità forse costituiscono un caso a parte. Se si dovesse per forza proporre un'ipotesi, direi che il contesto più vicino è - mutatis mutandis - quello di Naucrati (dono votivo).

62 Vd. n. 39. Come parallelo si pensi alla stele di Theodotos nel cui angolo sinistro superiore compare una sfinge che regge una piccola meridiana decorativa, cfr. Firatlı e Robert 1964, 33; Gibbs 1976,164 . Non è necessario, come sostiene Frratli, che l'orologio fosse un richiamo alla professione di filosofo del defunto, cfr., su questo esempio e in generale sul rapporto tra orologi e idea della morte, Bonnin 2013, 468-91, 481 per la stele di Theodotos.

63 Vd. n. 43.

64 Per Vilanova vd. n. 35.

65 Si veda Cavalier et al. 1992, 64-65; Dasen 2019. Ampia documentazione, sia bibliografica sia di materiali antichi, si trova nel sito del progetto Locus Ludi (https://locusludi.ch/). 
dire che se pure alcuni tra gli orologi miniatura che abbiamo sono stati dei giocattoli, è più probabile che questo si riferisca a quelli in materiali meno pregiati.

Un'altra possibilità che valorizza piuttosto la ricercatezza miniaturistica è riferire alcuni di questi orologi solari al contesto simposiale, per introdurre o accompagnare digressioni sul tempo, quasi novelli Atlanti che tengono in mano un oggetto di solito tutt'altro che maneggevole. ${ }^{67}$ L'indicazione I $\Sigma$ HMEPIA sul quadrante di Tanis, come anche la presenza delle linee zodiacali, avrebbe potuto favorire, ad esempio, una discussione su aspetti astronomici e temi connessi. Il paradosso di un orologio solare - non portatile - in avorio ci consente di immaginare l'aspetto performativo dietro l'oggetto: esso doveva essere messo in posizione in determinate occasioni, che avevano probabilmente un carattere sociale (ricevimento di ospiti) ed erano eventualmente animate da uno spirito ludico o (auto)celebrativo. Eventualmente avrebbe potuto anche giocare un ruolo alla conclusione del banchetto come dono ospitale, con richiamo al buon tempo trascorso assieme. Marziale non cita esplicitamente micro-orologi tra i possibili apophoreta, ma forse accanto alla serie di tavolette di cedro (14, 3: pugillares citrei) e a quelle di avorio (5: pugillares eborei), ai piccoli forzieri di avorio (12: loculi eborei) e di legno (13: loculi lignei), un ospite avrebbe potuto ben offrire un minuscolo orologio d'avorio o di pietra.

F.B.

\section{L'orologio solare e l'assetto residenziale tardo-repubblicano di piazza del Duomo}

Dal punto di vista archeologico, finora non disponevamo di indizi relativi al tenore sociale del quartiere residenziale pre-augusteo, mentre è ormai accertato e confermato anche da recenti interventi di scavo che la piazza del Duomo di Pisa per tutta l'età imperiale fu occupata da domus di alto livello, con pavimenti a mosaico e pareti affrescate. ${ }^{68}$

Sebbene da nuove ricerche sia possibile intravedere una connotazione analoga anche per l'assetto residenziale tardo-repubblicano, ${ }^{69}$ a mio avviso l'orologio solare costituisce un indizio decisivo in questa direzione. Infatti, a riprova dell'alto valore sociale, simbolico e allegorico che tale accessorio rivestiva nel mondo antico, si segnalano, tra $\mathrm{i}$ vari, ${ }^{70}$ tre esempi da dimore private del I sec. a.C. Si tratta di rappresentazioni di orologi solari nell'ambito dei cicli decorativi di residenze del comprensorio vesuviano: il noto mosaico dell'Accademia di Platone dalla villa di Titus Siminius Stephanus, in cui un quadrante conico si erge su un'alta colonna votiva al centro della scena, che raffigura i Sette Sapienti intenti a discutere in un paesaggio ideale; un affresco sulla parete ovest del peristilio della Casa della Fontana Piccola a Pompei, che rappresenta un quadrante sferico su una

67 Il topos di Atlante in questo contesto è abbastanza ovvio, per un esempio si veda l'orologio solare di Pompei in cui la griglia è retta da Atlante inginocchiato per lo sforzo (Dialface ID 631, Museo Archeologico Nazionale di Napoli, inv. 120464). Il proprietario della meridiana pisana avrebbe potuto maneggiarla con ben diversa facilità. Trimalcione aveva un orologio, probabilmente idraulico, nel suo triclinio (Petron., Sat. 26), cfr. Bonnin 2013, 471-73, Talbert 2017, 173, anche se rimane difficile valutare come interpretare la testimonianza in termini di scarto dalla norma ovvero di adesione a un uso comune. Considerazioni sulla percezione sociale degli orologi in Bonnin 2015, specialmente 309-49.

68 Alberti e Paribeni 2011, 24-25; Paribeni et al. 2011, 79-86.

69 Taccola 2020, 21, 27.

70 Presi in rassegna da Bonnin 2015, 309-49. 
colonna; ${ }^{71}$ infine, un affresco sulla parete sud del peristilio della villa di Publius Fannius Synistor a Boscoreale, in cui è raffigurato un globo coronato da uno gnomone e posizionato su un supporto cilindrico all'interno di un giardino: in quest'ultimo esempio, la rappresentazione del globo con gnomone è, significativamente, in diretto pendant con un "functioning sundial" rinvenuto nei pressi dell'affresco. ${ }^{72}$

I casi riportati richiamano chiaramente la funzione autocelebrativa in termini di ricchezza ed eleganza che i proprietari di tali residenze, aderendo più o meno genuinamente a un'élite colta e raffinata, intrisa del sapere filosofico greco, attribuivano a tali oggetti (e alle loro rappresentazioni), volutamente posizionati negli ambienti di massima rappresentanza di domus e ville. ${ }^{73}$

Tornando all'eccezionale esemplare pisano, la dimensione miniaturistica connota chiaramente il manufatto come accessorio personale, indicatore di status, appartenuto a un individuo di alto rango sociale, o comunque con disponibilità economiche tali da permettersi un articolo del genere da esibire nella propria residenza, prezioso di per sé e per il contenuto simbolico che veicolava, piuttosto che per il compito primario a cui doveva assolvere, la scansione delle ore diurne. A tal proposito, poiché il quadrante, pur aderendo a uno schema matematico, non avrebbe funzionato correttamente alla latitudine nella quale è stato rinvenuto (caratteristica che pare accomunare tutti gli esemplari miniaturistici noti), rimane il dubbio se il committente sia stato anche l'unico proprietario, ovvero, se l'orologio sia stato progettato appositamente per Pisa oppure sia finito secondariamente in possesso della persona che lo ha esposto e infine "perduto" a Pisa. Analogamente, è impossibile dire molto sull'identità e sulla provenienza dell'artefice dell'oggetto, se non riconoscerne l'abilità tecnica, né sapere se abbia lavorato proprio a Pisa. Benché l'unico confronto provenga dall'Egitto tolemaico e il materiale richiami da vicino il continente africano, non significa necessariamente, al netto delle affinità simboliche, che il quadrante di piazza del Duomo sia stato realizzato in un simile contesto. Infatti, rivolgendo e limitando lo sguardo all'Etruria settentrionale, indizi significativi quali la presenza di blocchetti grezzi in contesti tombali testimoniano che la lavorazione dell'avorio era praticata in loco già in età orientalizzante. $^{74}$

E.T.

71 Fröhlich 1997, 42, 94-5, figs. 248-49.

72 Bergmann 2013, 97, fig. 16. La studiosa segnala una rappresentazione simile, un globo su un vaso di metallo, su una parete nei pressi della piscina della villa cosiddetta di Poppea a Oplontis. In ogni caso, non è da escludere che l'affresco raffiguri una sfera armillare piuttosto che un orologio solare: secondo J. Bonnin vi sono troppe curve di declinazione e in più lo gnomone è posizionato verticalmente. Tuttavia, anche secondo lo studioso francese il dubbio rimane, potendo costituire la prima rappresentazione di un orologio solare, di un tipo noto attraverso rari confronti quali il globo di Prosymna (Dialface ID 284, Archaeological Museum of Nafplion, inv. $\lambda$ 2481) e la sfera di Matelica (Dialface ID 423, Museo Civico Archeologico di Matelica, inv. 77238).

73 Su questo aspetto cfr. Bonnin 2015, 198-202; Bonnin 2015, 212.

74 Cygielman 2010, 53 (Vetulonia, Circolo del Monile d'argento). Anche in questo caso, non è possibile specificare l'origine delle maestranze, anche se è verosimile che artisti del vicino Oriente, trasferiti in Italia, abbiano impiantato botteghe e formato artigiani locali. 


\section{Considerazioni conclusive}

L'orologio solare miniaturistico in avorio è un documento di straordinaria importanza non solo per le caratteristiche intrinseche (materiche e dimensionali), ma anche - e soprattutto - per le implicazioni socioculturali che il possesso e l'ostentazione di un manufatto del genere sottintendevano, come abbiamo mostrato attraverso un approccio olistico e necessariamente interdisciplinare che rendesse giustizia all'ampiezza concettuale di un oggetto così piccolo.

Grazie a esso possiamo intravedere in filigrana uno spaccato sociale di Pisa in età tardo-ellenistica (o tardo-repubblicana), periodo in cui la città etrusca, e ancora etruscofona alla metà del II secolo a.C., ${ }^{75}$ va realizzando in termini territoriali e giuridici il suo processo di romanizzazione.

Se i confronti stilistici non sono sufficienti a determinare una datazione precisa dell'oggetto, altre valutazioni, quali la diffusione degli orologi solari nel mondo italico, la giacitura stratigrafica e il momento di formazione e sviluppo del quartiere residenziale tardo-repubblicano, consentono di circoscriverne la cronologia tra il secondo quarto del II sec. a.C. e lo scorcio del I sec. a.C.

Malgrado le limitazioni emerse dallo studio gnomonico, questo manufatto singolare rappresentava una preziosa curiosità, enfatizzata dall'utilizzo dell'avorio di elefante, da esibire per connotare la ricchezza e l'eleganza del proprietario e per sottolinearne l'adesione, almeno formale, a una ristretta comunità colta e raffinata.

Pur non disponendo di informazioni più dettagliate sulla sua identità, possiamo immaginarlo come uno degli abitanti del quartiere residenziale di Piazza del Duomo, e avere una conferma del tenore sociale di quest'area della città in età tardo-repubblicana, che continuerà a essere occupata per tutta l'età imperiale da domus di alto livello.

E.T., F.B.

Riconoscimenti: L'indagine archeologica sistematica è stata eseguita dalla Soprintendenza Archeologica per la Toscana sotto la direzione di Adriano Maggiani in collaborazione con l'allora Istituto di Archeologia dell'Università di Pisa. Ringrazio calorosamente Claudia Rizzitelli, funzionario archeologo della Soprintendenza ABAP di Pisa e Livorno per aver concesso lo studio dei materiali recuperati nello scavo del saggio $\mathrm{D}$ in deposito presso il Laboratorio di Etruscologia e Archeologia Romana del Dipartimento di Civiltà e Forme del Sapere dell'Università di Pisa. Questo contributo si integra nel progetto di eccellenza del Dipartimento di Civiltà e Forme del Sapere (Università di Pisa) per il quinquennio 2018-22: I tempi delle strutture. Resilienze, accelerazioni e percezioni del cambiamento (nello spazio euro-mediterraneo).

\section{Riferimenti}

Adam, S. 2002. "Ancient sundials of Israel, part 1: Sundials found in Jerusalem." British Sundial Society Bulletin 14, no. 2: 52-57.

Alberti, A., e E. Paribeni. 2011. "Archeologia in Piazza dei Miracoli. Riflessioni a chiusura dei lavori per la pubblicazione." In Archeologia in Piazza dei Miracoli. Gli scavi 2003-2009, ed. E. Paribeni e A. Alberti, 17-30. Pisa: Felici. 
Ancient Sundials 2016= G. Graßhoff, E. Rinner, K. Schaldach, B. Fritsch, L. Taub, J. Sum, F. Kotschka. 2016. “Ancient sundials." Edition Topoi. DOI: 10.17171/1-1 (https://repository.edition-topoi.org/ collection/BSDP).

Bergmann, B. 2013. "Realia: Portable and painted objects from the Villa of Boscoreale." In La villa romaine de Boscoreale et ses fresques, ed. A. Barbet e A. Verbanck-Piérard, 79-103. Arles: Errance.

Bonnin, J. 2010. "Timekeepers in Britain, 43-780 AD. Origins, the Roman contribution, and Anglo-Saxon continuity." British Sundial Society Bulletin 22: 34-37.

Bonnin, J. 2013. "Horologia et memento mori... Les hommes, la mort et le temps dans l'Antiquité gréco-romaine." Latomus 72: 468-91.

Bonnin, J. 2015. La mesure du temps dans l'Antiquité. Paris: Les Belles Lettres.

Bonnin, J. 2020. "Der Meridian des Augustus in Rom." In Zeit in den Kulturen des Altertums: Antike Chronologie im Spiegel der Quellen, ed. R. Färber e R. Gautschy, 517-28. Wien, Köln, e Weimar: Böhlau Verlag.

Bruni, S. 2003. “Pisae." StEtr 69: 280-82.

Cantini, F., F. Fabiani, M. L. Gualandi e C. Rizzitelli, eds. 2020. Le case di Pisa. Edilizia privata tra Età romana e Medioevo. Atti del Convegno, Pisa, 2019. Firenze: All'insegna del Giglio.

Carreras, C., e E. Farré. In stampa. “Un model de rellotge de sol trobat a Iulia Libica (Llívia)." Revista d'Arqueologia de Ponent 31.

Cavalier, O., A. Durand, M. Manson e M. Rouvier-Jeanlin. 1991. “Jouets et jeux de l'enfance.” In Jouer dans l'Antiquité (cat. mostra, Marseille), Musée d'Archéologie Méditerranéenne - Centre de la Vielle Charité, 50-82. Marseille: Musées de Marseille; Paris: Réunion des Musées Nationaux.

Conspectus =E. Ettlinger, B. Hedinger, B. Hoffmann, P. M. Kenrick, G. Pucci, K. Roth-Rubi, G. Schneider, S. von Schnurbein, C. M. Wells e S. Zabehlicky-Scheffenegger, eds. 1990. Conspectus formarum terrae sigillatae italico modo confectae. Bonn: Habelt.

CVArr $^{2}=$ A. Oxé, H. Comfort e P. M. Kenrick, eds. 2000. Corpus Vasorum Arretinorum ${ }^{2}$ : A Catalogue of the Signatures, Shapes and Chronology of Italian Sigillata. Bonn: Habelt.

Cygielman, M. 2010. “Vetulonia: tra la prima età del ferro e l'Orientalizzante." In Signori di Maremma. Elites etrusche fra Populonia e Vulci (cat. mostra, Firenze), ed. M. Celuzza e G. C. Cianferoni, 4554. Firenze: Polistampa.

Dasen, V., ed. 2019. Ludique: Jouer dans l'Antiquité (cat. mostra, Lyon). Gent: Éditions Snoeck.

Evans, J. e M. Marée. 2008. "A miniature ivory sundial with equinox indicator from Ptolemaic Tanis, Egypt." Journal for the History of Astronomy 39: 1-17.

Filippetti, S. 2000. "La meridiana di Bevagna. Una nuova proposta di cronologia e di analisi." AnnPerugia 33: 49-64.

Firatlı N. e L. Robert. 1964. Les stèles funéraires de Byzance gréco-romaine. Paris: Maisonneuve.

Fröhlich, T. 1997. Casa della Fontana piccola (VI 8, 23. 24), Häuser in Pompeji 8. München: Hirmer.

Genovesi, S. 2014. "Nuovi bolli laterizi da Portus Pisanus e dal suo retroterra. Produzione e commercializzazione dei laterizi nell'Ager Pisanus, tra la metà del I secolo avanti Cristo e il I secolo dopo Cristo." Rassegna di Archeologia 24/B: 45-84.

Gibbs, S. L. 1976. Greek and Roman Sundials. New Haven e London: Yale University Press.

Gliozzo, E., D. Manacorda e E. J. Shepherd. 2004. "I bolli volvs nell'Etruria romana: tipologia e problemi di interpretazione." In Materiali per Populonia 3, ed. M. L. Gualandi e C. Mascione, 191-216. Firenze: All'Insegna del Giglio.

Gumà i Marquès, M. M. 1992. “Un rellotge de sol d'època republicana trobat a Darró.” Miscellània Penedesenca 16: 145-57.

Harris, W. V. 1971. Rome in Etruria and Umbria. Oxford: Clarendon Press.

Heinemann, A. 2021. "Skin and bone. Lala of Cyzicus and the softness of ivory." In The Nature of Art. Pliny the Elder on Materials, ed. A. Anguissola e A. Grüner. Turnhout: Brepols Publishers.

Jones, A. 2020. "Greco-Roman sundials: Precision and displacement." In Down to the Hour: Short Time in the Ancient Mediterranean and Near East, ed. K. J. Miller e S. Symons, 125-57. Leiden: Brill.

Lapatin, K. 2001. Chryselephantine Statuary in the Ancient Mediterranean World. Oxford: Oxford University Press.

Locher, K. 1993. “Two Greco-Roman sundials from Alexandria and Dion." Journal for the History of Astronomy 24.4: 300-2.

Lo Sardo, E., ed. 2005. Eureka! Il genio degli antichi (cat. mostra, Napoli). Napoli: Electa. 


\section{Un orologio solare miniaturistico da Pisa}

Macalister, R. A. S. e J. Garrow Duncan. 1926. Excavations on the Hill of Ophel, Jerusalem, 1923-1925. London: Palestine Exploration Fund.

Paribeni, E., L. Parodi, S. Genovesi, F. Mani e D. Stiaffini. 2011. “Un quartiere residenziale di Pisa romana (Periodo II)." In Archeologia in Piazza dei Miracoli. Gli scavi 2003-2009, ed. E. Paribeni e A. Alberti, 71-166. Pisa: Felici editore.

Pasquinucci, M. 1995. "Colonia Opsequens Iulia Pisana: qualche riflessione sulla città ed il suo territorio." AnnPisa 25: 310-17.

Petrain, D. 2014. Homer in Stone: The "Tabulae Iliacae" in Their Roman Context. Cambridge: Cambridge University Press.

Petrie, W. M. F. 1886. Naukratis. Part I, 1884-85. London: Trübner and Co.

Rizzitelli, C. e F. Fabiani. 2020. "Area dell'Arcivescovado. La ricomposizione di contesti residenziali da vecchi e nuovi scavi." In Le case di Pisa. Edilizia privata tra età romana e Medioevo, ed. F. Cantini, F. Fabiani, M. L. Gualandi e C. Rizzitelli, 39-48. Firenze: All'Insegna del Giglio.

Sangriso, P. 1999. "La data delle colonie triumvirali di Luni e di Pisa. Note su CIL XI 1330." Epigraphica 61: 47-50.

Savoie, D. 1989. "Les dates des quatre saisons." Observations et Travaux 19: 3-6.

Savoie, D. 2020. "Analyse gnomonique d'un cadran solaire antique." Cadran Info 41: 166-79.

Squire, M. J. 2011. The Iliad in a Nutshell: Visualizing Epic on the Tabulae Iliacae. Oxford e New York: Oxford University Press.

Stewart, S. 1993. On Longing: Narratives of the Miniature, the Gigantic, the Souvenir, the Collection. Durham, NC, e London: Duke University Press.

Taccola, E. 2019. Uno sguardo su Pisa ellenistica da piazza del Duomo. Lo scavo del saggio D 1985-1988. Oxford: Archaeopress.

Taccola, E. 2020. "La nascita di un quartiere residenziale: le domus ai piedi della Torre tra II e I secolo a.C." In Le case di Pisa. Edilizia privata tra età romana e Medioevo, ed. F. Cantini, F. Fabiani, M. L. Gualandi e C. Rizzitelli, 17-28. Firenze: All'insegna del Giglio.

Talbert, R. J. A. 2017. Roman Portable Sundials: The Empire in Your Hand. New York: Oxford University Press.

Taylor, M. V. e R. G. Collingwood. 1926. “Roman Britain in 1926.” JRS 16: 216-44. 